\title{
Combined perfluoropropane gas and viscoelastic material injection for anterior chamber reformation following trabeculectomy
}

\author{
S Kurtz, I Leibovitch
}

Br J Ophthalmol 2002;86:1225-1227

Aim: To describe a novel technique to treat hypotony and shallow anterior chamber following trabeculectomy

Methods: Three patients who underwent fornix based trabeculectomy with mitomycin $C(M M C)$ presented in the postoperative days with a shallow anterior chamber, hypotony, and overfiltrating blebs. They were treated with an injection of $0.2 \mathrm{ml}$ of $16 \%$ perfluoropropane $\left(\mathrm{C}_{3} \mathrm{~F}_{8}\right)$ to the anterior chamber followed by an injection of a viscoelastic material.

Results: In all patients the anterior chamber was reformed successfully without any complications.

Conclusions: Patients with a shallow anterior chamber and overfiltrating blebs following a recent trabeculectomy can be treated successfully with a combination of a long acting gas and a viscoelastic material.

$\mathrm{T}$ rabeculectomy is still the most common filtering procedure for the treatment of uncontrolled glaucoma in patients on maximal tolerated medical therapy. The prognosis varies according to patient characteristics, the type of glaucoma, and the type of filtering procedure. Adjunctive treatment with intraoperative antifibrosis drugs has become very popular within the past 10 years not only in eyes at high risk for trabeculectomy failure, but also in low risk eyes undergoing primary trabeculectomy. The results show a higher success rate and a lower mean postoperative intraocular pressure (IOP) than trabeculectomy without these adjunctive drugs. ${ }^{12}$

One of the common complications following the filtering procedure is hypotony with or without shallowing of the anterior chamber. This might cause corneal decompensation, progression of cataract in phakic eyes, and reduction of visual acuity because of choroidal folds and hypotonic maculopathy. ${ }^{3-5}$ Surgical anterior chamber reformation may be required if other methods such as cycloplegics, patching, or bandage contact lenses are not effective.

Anterior chamber reformation could be performed using air, ${ }^{67}$ viscoelastic material, ${ }^{8}$ or perfluorocarbone gases. ${ }^{9-12}$ Each of these methods has possible complications, limiting their use especially in phakic patients. Injection of expansile concentrations of anterior chamber perfluorocarbone gases was previously complicated by IOP spikes, and cataract formation if the gas comes in contact with the lens in phakic eyes.

We present a novel technique combining the injection of perfluoropropane $\left(\mathrm{C}_{3} \mathrm{~F}_{8}\right)$ and hyaluronic acid for hypotony and shallow anterior chamber following trabeculectomy. By using a non-expansile concentration of perfluorocarbone gases we could reduce the possible complications of IOP spikes and cataract formation, making this method a possible alternative applicable to all patients, as well as phakic ones.

\section{PATIENTS AND METHODS}

Three patients were included in the study. All patients underwent fornix based trabeculectomy with mitomycin C (MMC) $0.2 \mathrm{mg} / \mathrm{ml}$ for 2 minutes and presented in the postoperative days with hypotony and a flat anterior chamber. A $0.2 \mathrm{ml}$ volume of $16 \% \mathrm{C}_{3} \mathrm{~F}_{8}$ gas mixed with room air was injected into the anterior chamber using a 30 gauge angled cannula through the paracentesis site of the filter operation, followed by injection of $1 \mathrm{ml}$ of a medium heavy viscoelastic material containing sodium hyaluronate, $12 \mathrm{mg} / \mathrm{ml}$ (Biolon Prime, BioTechnology General, Israel) at the same site. Clinical details of the patients are presented in the results section.

\section{RESULTS \\ Patient 1}

A 64 year old woman with primary open angle glaucoma (POAG) in her right eye was admitted with uncontrolled IOP (27 mm Hg) and progression of her visual field defect. Best corrected visual acuity before operation was 20/30. The anterior segment was unremarkable with an open wide angle and no cataract. The optic nerve cup to disc ratio was 0.7 , with an inferior rim notching and the macula was normal.

She underwent fornix based trabeculectomy with MMC in her right eye as described above in the methods section. On the first postoperative day the patient was treated with topical cyclopentolate and phenylephrine hydrochloride every 6 hours. On the second postoperative day her visual acuity decreased to 20/200, the bleb was highly elevated and with no leak (confirmed using a fluorescein strip). IOP was $2 \mathrm{~mm} \mathrm{Hg}$, the cornea was clear with descemet folds, and the anterior chamber was very shallow (one corneal thickness). The optic disc was mildly engorged and the retina was attached without ciliochoroidal detachment. Despite repeated injections of a heavy viscoelastic material, the anterior chamber remained very shallow. A $0.2 \mathrm{ml}$ volume of $16 \% \mathrm{C}_{3} \mathrm{~F}_{8}$ gas mixed with air was injected into the anterior chamber, followed by injection of $1 \mathrm{ml}$ of a viscoelastic material, as described above, until the anterior chamber became deep. The patient was instructed to remain in the upright position as much as possible. During the next week the anterior chamber remained deep, the IOP ranged between 10-14 $\mathrm{mm} \mathrm{Hg}$, and the filtering bleb was mildly elevated. Ultrasound biomicroscopy (UBM) performed after the procedure did not reveal signs of peripheral choroidal detachment. The gas bubble gradually disappeared after 12 days. Six weeks later, the IOP was $12 \mathrm{~mm} \mathrm{Hg}$ without any medications. The anterior chamber was deep and quiet and the filtering bleb was avascular and moderately elevated. Visual acuity returned to 20/30, the gas bubble was not seen, and there were no signs of cataract formation or other side effects. There was no change after 3 more months of follow up.

\section{Patient 2}

A 45 year old man with juvenile onset open angle glaucoma was admitted because of advanced damage to the optic nerve 
and visual field deterioration in his right eye, despite maximal tolerated medical therapy.

Best corrected visual acuity in the right eye was 20/40 and IOP was $23 \mathrm{~mm} \mathrm{Hg}$.

The anterior chamber was normal without cataract and the anterior chamber angle was widely open with prominent iris processes. The optic nerve cup to disc ratio was 0.9 with a pale rim, and the macula was normal. Visual field analysis of the right eye showed a deep nasal step with double arcuate scotomas.

The patient underwent trabeculectomy with MMC as described earlier. On the first postoperative day, visual acuity was 20/400, the IOP was $2 \mathrm{~mm} \mathrm{Hg}$, with no leakage. The bleb was highly elevated, the cornea was clear and the anterior chamber was shallow ( 0.5 corneal thickness in the centre with irido-corneal touch in the nasal and temporal periphery). The patient was treated with topical cyclopentolate and phenylephrine hydrochloride every 6 hours. On the next day, injection of viscoelastic material had a transient effect lasting only a few hours and the anterior chamber remained very shallow. $\mathrm{C}_{3} \mathrm{~F}_{8}$ gas was injected, followed by a viscoelastic material, as described above. During the following week, the anterior chamber remained deep with an IOP of 8-10 $\mathrm{mm} \mathrm{Hg}$. The gas bubble gradually disappeared after 12 days After 6 weeks visual acuity was 20/50, The IOP was $14 \mathrm{~mm} \mathrm{Hg}$ without any medications. The anterior chamber was deep without a gas bubble and the filtering bleb was highly elevated with microcystic changes. No cataract formation or other complications were noted. There was no change after 3 more months of follow up.

\section{Patient 3}

A 52 year old woman was admitted because of normal tension glaucoma in her left eye with advanced disc and visual field damage despite maximal tolerated medical therapy. Best corrected visual acuity was 20/50 in her left eye and IOP was 19 $\mathrm{mm} \mathrm{Hg}$. The anterior chamber was deep and quiet with a widely open anterior chamber angle. The optic nerve head showed a pale thin rim with almost total cup to disc ratio. The visual field showed deep nasal and paracentral scotoma, threatening fixation.

She underwent trabeculectomy with MMC as described earlier. On the first postoperative day the patient was treated with topical cyclopentolate and phenylephrine hydrochloride every 6 hours. On the second postoperative day the visual acuity deteriorated to finger counting. The IOP was $1-2 \mathrm{~mm} \mathrm{Hg}$, the anterior chamber was shallow (one corneal thickness) without irido-corneal touch but with a hyphaema of $0.5 \mathrm{~mm}$. The cornea was clear with prominent descemet folds and the bleb was highly elevated without any leakage. The retina was attached with peripheral nasal and superior choroidal detachment.

Injection of a viscoelastic material had a temporary effect lasting 12 hours. $\mathrm{C}_{3} \mathrm{~F}_{8}$ was injected in to the anterior chamber followed by viscoelastic injection as described above. In the next 2 weeks of follow up, IOP was between 4 and $10 \mathrm{~mm} \mathrm{Hg}$. The gas bubble gradually disappeared after 14 days. After 6 weeks, visual acuity was 20/50, IOP was $8 \mathrm{~mm} \mathrm{Hg}$ without medications, and the filtering bleb was highly elevated and avascular. The anterior chamber was deep and quiet and the lens was clear. The retina was attached without choroidal detachments. After 3 more months of follow up visual acuity was $20 / 40$, with no other clinical changes on examination.

\section{DISCUSSION}

Hypotony and shallow anterior chamber in the immediate postoperative period after trabeculectomy might result for four main reasons: a leaking bleb, overfiltration of the bleb, the use of concomitant drugs which might cause hypotony, and a cilio-choroidal detachment.
Our patients were diagnosed has having overfiltration because there were no signs of leakage and they did not use any hypotensive drugs. In the first two patients there were no signs of choroidal detachment and in the third case the detachment was too small to cause this complication and it was probably secondary to the hypotony. The appearance of high blebs with shallow anterior chambers with very low IOPs and no prominent choroidal detachment made the diagnosis of overfiltration. This complication is usually caused by improper closure of the scleral wound or because of the use of antifibrosis agents like MMC. Hypotony is also more common in young patients as in the second case and in myopic patients as in the third case.

Injection of a heavy viscoelastic material resulted in a temporary and transient effect, because of the high filtration rate. Some glaucoma surgeons do not intervene with anterior chamber deepening manoeuvres in the first postoperative days if there is not lens-cornea touch, as this situation may reverse itself, and they only treat these patients with cycloplegic drugs.

Other possible treatments, mainly for managing bleb leaks, include medical treatment to decrease aqueous humour formation, pressure patching, ${ }^{13}$ conjunctival suturing, ${ }^{14}$ oversized contact lens, ${ }^{15}$ and more recently autologous blood injections. ${ }^{16-18}$ These measures have variable success rates, some with a transitory effect, necessitating revision of the scleral flap.

There were reports on anterior chamber reformation using air. ${ }^{67}$ Unfortunately, air could not be controlled and it often escapes out of the chamber into the bleb. Additionally, air may cause endothelial cell loss. ${ }^{19}$ Injection of viscoelastic materials such as hyaluronic acid was also reported. ${ }^{8}$ The main disadvantage of this method is the significant rise in IOP as a result of the large amount of material injected. Hyaluronic acid mixes with the aqueous fluid and is cleared from the eye in the aqueous outflow, and therefore its effect is short and temporary.

Injection of longstanding and expansile gases (used in retinal surgery for internal tamponade of retinal breaks) for reformation of the anterior chamber following glaucoma surgery was previously reported. ${ }^{9-12}$ The main gases used are sulphur hexafluoride $\left(\mathrm{SF}_{6}\right)$ and perfluoropropane $\left(\mathrm{C}_{3} \mathrm{~F}_{8}\right)$. These gases are not water soluble and expand at high concentrations in an aqueous medium. ${ }^{20}$ The high surface tension of these gases and the single bubble that is formed help in sealing the fistula and reforming the anterior chamber.

These gases were proved to be inert in the vitreous cavity and non-toxic to the retina. The main reported intraocular side effect of these gases was lens opacities at the area of lensgas contact. ${ }^{10}$ Studies in rabbit and cat eyes have demonstrated corneal oedema due to endothelial dysfunction after an injection of pure $\mathrm{C}_{3} \mathrm{~F}_{8}$ and $\mathrm{SF}_{6}{ }^{21}$ When lower concentrations of these gases were used, only swollen endothelial cells were seen. ${ }^{22}$ These corneal complication were neither observed in the reports mentioned, ${ }^{9-12}$ nor in our patients.

In our patients, we decided to continue to anterior chamber reformation on the second day of cycloplegic treatment because of the significant shallow chambers and the possible risks of lens-cornea touch, anterior synechiae, choroidal detachment, hypotony maculopathy, and bleb failure.

In this case series we present a simple, easy and short technique, which can be performed as an office procedure while the patient is seated at the slit lamp and without complications. The major problem with this procedure is the risk of endophthalmitis, especially if the procedure is done at the slit lamp and not in the operating room. We believe that this procedure is successful because the gas bubble, which can remain in the eye for up to 3 weeks, blocks the internal ostium of the sclerostomy, allowing the wound to heal. This block is probably not complete because there were no pressure spikes during our close follow up, and this tamponade, with the aid of 
viscoelastic material, allowed continuous reformation of the anterior chamber.

As gases are injected in a non-expendable concentration, the risk of high IOP spikes is low. The combination with the viscoelastic material allows injection of a smaller amount of gas, thereby reducing the risk of lens contact and cataract formation. The existence of the gas permits using a smaller amount of viscoelastic material and keeps it for a longer time in the anterior chamber without extensive IOP elevation.

$\mathrm{C}_{3} \mathrm{~F}_{8}$ was used in anterior segment surgery not only for anterior chamber reformation but also as a successful treatment for descemet detachment after cataract surgery, ${ }^{23-25}$ and experimentally injected to the subconjunctival area to enhance the results of filtering procedures. ${ }^{26}$ The procedure we described here is another way of using this gas combined with a viscoelastic material to aid coping with one of the severe complications in glaucoma filtration surgery.

A prospective randomised study is needed to further evaluate the usefulness of this method in managing hypotony and shallow anterior chamber following trabeculectomy.

\section{Authors' affiliations}

S Kurtz, I Leibovitch, Department of Ophthalmology, Tel-Aviv Sourasky Medical Center, Sackler Faculty of Medicine, Tel-Aviv University, Tel-Aviv, Israel

Correspondence to: Dr lgal Leibovitch; lleibo@bezeqint.net

Accepted for publication 9 May 2002

\section{REFERENCES}

1 Chen CW, Huang HT, Bair JS, Lee CC. Trabeculectomy with simultaneous application of mitomycin-C in refractory glaucoma. J Ocul Pharmacol 1990;6:175-82

2 Palmer SS. Mitomycin as adjunct chemotherapy with trabeculectomy. Ophthalmology 1991;98:317-21.

3 Zacharia PT, Deppermann SR, Schuman JS. Ocular hypotony after trabeculectomy with mitomycin C. Am J Ophthalmol 1993;116:314-26.

4 Costa VP, Wilson RP, Moster MR, et al. Hypotony maculopathy following the use of topical mitomycin $\mathrm{C}$ in glaucoma filtration surgery. Ophthalmic Surg 1993;24:389-94.

5 Shin DH. Management of flat anterior chamber with hypotonia after glaucoma surgery. Glaucoma 1982;4:193-7.
6 Srewart RH, Kimbrough RL. A method of managing flat anterior chamber after trabeculectomy. Ophthalmic Surg 1980;11:382-3.

7 Fourman S. Management of cornea-lens touch after filtering surgery for glaucoma. Ophthalmology 1990;97:424-8.

8 Gerber SL, Cantor LB. Slit lamp reformation of the anterior chamber following trabeculectomy. Ophthalmic Surg 1990;21:404-40.

9 Franks WA, Hitchings RA. Injection of perfluoropropane gas to prevent hypotony in eyes undergoing tube implant surgery. Ophthalmology 1990;97:899-903.

10 Franks WA, Hitchings RA. Intraocular gas injection in the treatment of cornea- lens touch and choroidal effusion following fistulizing surgery. Ophthalmic Surg 1990;21:831-4.

11 Beigi B, O'Keefe M, Algawi K, et al. Sulphur hexafluoride in the treatment of flat anterior chamber following trabeculectomy. Eye $1997 ; 11: 672-6$.

12 Weinstein 0 , Oshry T, Tessler Z, et al. Use of sulphur hexafluoride for anterior chamber reformation following trabeculectomy. $\mathrm{Br} J \mathrm{Ophthalmol}$ 1998;82:1220.

13 Mattox C. Management of the leaking bleb. J Glaucoma 1995;4:370-4.

14 Tomlinson CP, Belcher CD, Smith PD, et al. Management of leaking filtration blebs. Ann Ophthalmol 1987;19:408-1 1.

15 Blok MD, Kok JH, van Mil C, et al. Use of the megasoft bandage lens for treatment of complications after trabeculectomy. Am J Ophthalmol 1990; 1 10:264-8.

16 Wise JB. Treatment of chronic postfiltration hypotony by intrableb injection of autologous blood. Arch Ophthalmol 1993;111:827-30.

17 Leen MM, Moster MR, Katz L, et al. Management of overfiltering and leaking blebs with autologous blood injection. Arch Ophthalmol 1995; 113:1050-5.

18 Choudhri SA, Herndon LW, Damji KF, et al. Efficacy of autologous blood injection for treating overfiltering or leaking blebs after glaucoma surgery. Am J Ophthalmol 1997; 123:554-5.

19 Olson RJ. Air and the corneal endothelium. Arch Ophthalmol 1980;98: 1283-4

20 Lincoff $A$, Haft D, Liggett $P$, et al. Intravitreal expansion of perfluorocarbon bubbles. Arch Ophthalmol 1980;98:1646.

21 Foulks GN, de Juan E, Hatchell DL, et al. The effect of perfluoropropane on the cornea in rabbits and cats. Arch Ophthalmol 1987;105:256-9.

22 Lee DA, Wilson MR, Yoshizumi MO, et al. The ocular effects of gases when injected into the anterior chamber of rabbit eyes. Arch Ophthalmol $1991 ; 109: 571-5$.

23 Macsai MS, Gainer KM, Chisholm L. Repair of Descemet's membrane detachment with perfluoropropane $\left(C_{3} F_{3}\right)$. Cornea 1998:17:129-34.

24 Kremer I, Stiebel H, Yassur Y, et al. Sulfur hexafluoride injection for Descemet's membrane detachment in cataract surgery. J Cataract Refract Surg 1997;23:1449-53

25 Mahmood MA, Teichmann KD, Tomey KF, et al. Detachment of Descemet's membrane. J Cataract Refract Surg 1998;24:827-33.

26 Tym WH, Seah SKL. Augmentation of filtering blebs with perfluoropropane gas bubble. Ophthalmology 1999;106:545-9. 